\title{
Pedagogía Universitaria en contexto de pandemia. Relatos docentes sobre prácticas de enseñanza y de evaluación en el ingreso y el primer año
}

\section{University pedagogy in a pandemic context. Professors stories on teaching practices and evaluation in the admission and the first year}

\author{
María Verónica Cheli \\ https://orcid.org/0000-0003-3528-0498 \\ unlp.veronicacheli@gmail.com \\ Facultad de Humanidades y Ciencias de la \\ Educación | Instituto de Investigaciones en \\ Humanidades y Ciencias Sociales (IdIHCS) \\ CONICET-UNLP | Argentina
}

\author{
Jesica Montenegro \\ https://orcid.org/0000-0003-3264-249X \\ montenegro_jessica@yahoo.com.ar \\ Facultad de Humanidades y Ciencias de la \\ Educación | Instituto de Investigaciones en \\ Humanidades y Ciencias Sociales (IdIHCS) \\ CONICET-UNLP | Argentina
}

\author{
María Gabriela Marano \\ https://orcid.org/0000-0002-9201-3946 \\ gabimarano@gmail.com \\ Facultad de Humanidades y Ciencias de la \\ Educación | Instituto de Investigaciones en \\ Humanidades y Ciencias Sociales (IdIHCS) \\ CONICET UNLP | Argentina
}

\section{RESUMEN}

El trabajo trata sobre la sistematización de los relatos de docentes universitarias/ os acerca de la enseñanza en el tramo inicial de distintas carreras de la Universidad Nacional de La Plata en contexto de pandemia. Corresponde a las expresiones de las y los docentes cursantes de dos Seminarios de posgrado referidos a la democratización de la formación universitaria y a las prácticas pedagógicas en el ingreso y primer año.

Se analizan las problematizaciones que las y los profesoras/es realizaron acerca de la reconfiguración de sus propias prácticas de enseñanza y de evaluación en el contexto particular de virtualidad forzada. Las fuentes de acceso a la información son las expresiones producidas en los diversos dispositivos implementados en entornos virtuales de los cursos mencionados. Las reflexiones que arribamos dan el guiño para poner en tensión las prácticas docentes universitarias con las trayectorias estudiantiles y abren el debate sobre la pedagogía universitaria basada en un enfoque de derechos.

PALABRAS CLAVE

docencia universitaria, relatos docentes, democratización, derecho a la educación superior. 
KEY WORDS

university teaching, teaching experiences reports,

democratization,

right to higher education.

\section{ABSTRACT}

This paper addresses the systematization of the stories of professors about teaching in the initial section of different careers at the Universidad Nacional de La Plata in the context of a pandemic. It corresponds to the expressions of the professors attending two postgraduate seminars referring to the democratization of university education and pedagogical practices in entry and first year.

The problematizations that the professors made about their own teaching and evaluation practices in the particular context of forced virtuality are analyzed. The sources of access to the information are the produced expressions in the different devices implemented in virtual environments of the mentioned courses. The conclusions we have arrived, allow us to put university teaching practices with student trajectories in tension and open the debate on university pedagogy based on a rights-based approach. 


\section{PRESENTACIÓN}

En este trabajo se presenta una sistematización de los relatos de algunas/os docentes universitarias/os acerca de la docencia y la enseñanza en el tramo inicial de distintas carreras de la Universidad Nacional de La Plata (UNLP) en contexto de pandemia. Esta memoria corresponde a las expresiones de las y los docentes cursantes de dos Seminarios de posgrado referidos a la democratización de la formación universitaria y a las prácticas pedagógicas en el ingreso y primer año, destinados a profesores de la UNLP².

Las fuentes de acceso a la información son las expresiones producidas en los diversos dispositivos implementados en entornos virtuales de los cursos mencionados: wikis, foros, muros colaborativos, encuentros sincrónicos y tareas, donde las y los docentes problematizaron sobre diversos aspectos, entre los que seleccionamos la enseñanza y la evaluación; todo ello en relación con las trayectorias estudiantiles de las y los ingresantes, y el derecho a la educación superior. A partir de ellas, analizamos las problematizaciones que realizaron las/os docentes acerca de sus propias prácticas docentes, en el contexto particular del aislamiento social preventivo y obligatorio.

\section{RECONFIGURACIONES ACERCA DE LA ENSEÑANZA Y LA EVALUACIÓN EN PRIMER AÑO UNIVERSITARIO EN CONTEXTO DE PANDEMIA}

\section{RELATOS ACERCA DE LA ENSEÑANZA}

Las exigencias del contexto de emergencia sanitaria plantearon una redefinición en la tarea de enseñar y las nuevas configuraciones del "aula" o de la "clase" interpelaron a los/as docentes en sus significados y efectos ${ }^{3}$.

2 El primer Seminario se tituló "La universidad como derecho: los desafíos de las prácticas docentes en los primeros años" y fue ofrecido en el Programa de Capacitación Docente UNLP-ADULP. El segundo se denominó "La democratización en la universidad: perspectivas político-pedagógicas para pensar el ingreso y el primer año", perteneciente a la carrera de Especialización en Docencia Universitaria de la UNLP. Ambos se desarrollaron en forma virtual (plataforma institucional de la UNLP) durante el primer y segundo cuatrimestre del año 2020, respectivamente.

3 Los discursos de los/as docentes sobre la enseñanza en tiempo de pandemia se recuperaron de una tarea en la que establecieron dificultades, problemas, desafíos que se presentaron en las prácticas docentes e institucionales que inciden en la permanencia de los/as estudiantes. 
Uno de los desafíos estuvo relacionado con el "pasaje" de la propuesta de la enseñanza presencial a la virtual. Por la rapidez con la que se suscitaron los cambios -especialmente en las materias del primer cuatrimestre -, una de las primeras definiciones, casi por inercia, fue la de trasladar o "trasplantar" las propuestas presenciales a la virtualidad. Sin embargo, en ese tránsito, algunos/as docentes fueron advirtiendo que las nuevas condiciones de enseñanza requerían de cambios y adecuaciones. En definitiva, el desafío consistía en elaborar una propuesta que contemplara las particularidades del contexto de la enseñanza virtual y de un contexto social profundamente expulsivo, por rasgos que son estructurales y que la pandemia los hizo más evidentes. De allí que para estos docentes fue necesario recrear la propuesta de enseñanza, y la pregunta por el cómo enseñar en la virtualidad se convirtió en el principal desafío: "...los docentes y estudiantes nos vimos frente a una nueva dificultad, la enseñanza y el aprendizaje virtual" (docente de Programación Informática). Aquellos/as docentes que no sortearon ese pasaje o esa traducción a lo virtual advirtieron que no fue favorable para la permanencia de sus estudiantes:"(...) he notado que en muchos cursos se llevó lo presencial a lo virtual con alguna que otra implementación de actividades (...). Esto sumado a factores económicos podría actuar como un factor de desmotivación a la hora de decidir continuar con la carrera" (docente de Análisis Clínicos Veterinarios). Aquellos/as profesores/as que establecieron cambios en la enseñanza hicieron mención centralmente a la creación de recursos didácticos digitales (grabación de videos, escritura de clases, apuntes, gráficos, elaboración de power point, formulación de casos clínicos, entre otros.). En sus descripciones se reconoce que estas producciones fueron definidas con relación a los contenidos, los propósitos, las particularidades de los estudiantes y el contexto en el que se dio la experiencia.

Como sabemos, la enseñanza es en esencia un acto de comunicación (Juarros y Levy, 2020). Por ello, otra de las preocupaciones de algunos/as docentes estuvo vinculada con cómo establecer el vínculo pedagógico, cómo lograr formas de vinculación y comunicación con sus estudiantes a través de los entornos virtuales: "La idea era mantener de la mejor manera posible el contacto con las/os estudiantes, tratar de lograr crear un ambiente que les sirva de sostén, donde se pueda establecer ese ida y vuelta entre docentes y alumnos y entre el grupo de pares entre sí" (docente de Derecho Romano). A partir de la necesidad de "vernos las caras", se desplegaron disímiles canales de comunicación que permitieron desarrollar cierta interactividad en la comunicación, tales como: encuentros sincrónicos, videos de presentación temática, videoconferencias, videollamadas, salas de Facebook, chats, etc.

En los discursos de los/as profesoras, ese "pasaje" de lo presencial a lo virtual no se produjo sin brechas digitales (Gómez, 2020). Desde sus perspectivas, ello se manifiesta en las desiguales posibilidades de conectividad y accesibilidad de los/as estudiantes, que tiene su co- 
rrelato con las desigualdades socioeconómicas y de infraestructura en sociedades como las nuestras: "aproximadamente un tercio de los alumnos no tienen $P C$, no saben usarla, no están en condiciones de pagar un servicio de internet (docente de Programación Informática). Estas desigualdades materiales se reconocieron como parte de los procesos de deserción/exclusión en el contexto de la pandemia y el aislamiento obligatorio: "(...) con la pandemia pude notar que existe una brecha económica y de alcances tecnológicos que dificultan el aprendizaje de los alumnos". Otros/as identifican que a esas desigualdades materiales se les sumaron las desigualdades educativas existentes: "A los problemas de acceso a una computadora, a la conectividad, se le agregaron serias dificultades en la comprensión de los textos" (docentes de Obstetricia Patológica). Otras perspectivas reconocen estas desigualdades educativas previas a la pandemia y que continúan presentes: "en la virtualidad se siguen apreciando las problemáticas típicas del primer año, como ser la falta de habilidades y hábitos académicos" (docente de la Facultad de Ciencias Jurídicas y Sociales).

De acuerdo con las perspectivas relevadas, pudimos reconocer que, en este escenario mediado tecnológicamente, el desafío pedagógico abarcó no sólo transformaciones del espacio y del tiempo, sino también la reorganización de los saberes y la redefinición de la comunicación y de las relaciones de autoridad.

De acuerdo con las perspectivas relevadas, pudimos reconocer que, en este escenario mediado tecnológicamente, el desafío pedagógico abarcó no sólo transformaciones del espacio y del tiempo, sino también la reorganización de los saberes y la redefinición de la comunicación y de las relaciones de autoridad.

Otro aspecto importante tuvo que ver con la adquisición de prácticas de lectura y escritura de género académico. Como es sabido, las dificultades que los/as ingresantes presentan a la hora de enfrentarse a los textos académicos se debe en parte a cómo los/as profesores/as reciben a los recién llegados y comparten la cultura académica que ellos/as han adquirido como miembros de una comunidad disciplinar. En los Seminarios brindados, se trabajó esta dimensión, proponiendoles la realización de una wiki sobre las prácticas de alfabetización académica implementadas y cómo podrían ampliarse y mejorarse.

Las y los profesoras/es cursantes que dan clases en primer año se dividieron entre las/os que manifestaron que implementaron estrategias y aquellos que dijeron no haberlo hecho.

Las/os docentes que realizaron una propuesta sistemática de lectura y escritura con evaluación y retroalimentación, propusieron tareas como: relacionar el/la autor/a con su contexto, trabajar sobre el proceso de interpretación, proponer escrituras parciales, etc. En todos estos casos manifestaron estar satisfechas/os con los resultados, en la medida que las/os estudiantes se comprometieron con las actividades: "los resultados fueron buenos de los que entregaban todos los trabajos 
prácticos" (docente de Filosofía). También se marcó que la virtualidad condiciona las propuestas: "Los resultados en general han sido buenos, destaco la dificultad de poder realizar actividades más complejas en el contexto particular de la pandemia" (docente de Psicología). Este tipo de tarea -que requiere acuerdos didácticos-, muchas veces queda librada a las iniciativas individuales de las/os profesoras/es.-"No existe acuerdo colectivo..., cada docente aborda la enseñanza de la materia desde la experiencia individual" (docente de Introducción a la Química). La educación en "virtualidad obligada" generó gran impulso por la innovación pedagógica-didáctica. La estrategia más desarrollada fue la producción de materiales de enseñanza con pistas de lectura para la apropiación de los textos. Las cátedras se esforzaron en elaborar materiales para favorecer la comprensión, con predominio de guías de lectura, materiales escritos y piezas audiovisuales generados por el propio equipo docente, además de la selección y curaduría de materiales y recursos externos.

\section{De acuerdo con las perspectivas relevadas, pudimos reconocer que, en este escenario mediado tecnológicamente, el desafío pedagógico abarcó no sólo transformaciones del espacio y del tiempo, sino también la reorganización de los saberes y la re- definición de la comunicación y de las relaciones de autoridad.}

Por su parte, los/as docentes que no trabajaron el tema, igualmente reconocieron la problemática: "No existe práctica de alfabetización académica y se nota el problema de interpretación de enunciados" (docente de Programación Informática). De todos modos, en algunos casos, este reconocimiento percibe la alfabetización académica como un añadido complementario y no como un objeto de enseñanza que hace a la comprensión de la misma disciplina: "No existe trabajo detenido por la premura en avanzar y dar los contenidos" (docente de Introducción a la Filosofía)

Los planteos actuales distinguen entre alfabetización (proceso de enseñanza para favorecer el acceso a las culturas escritas de las disciplinas) y literacidad/es, (prácticas culturales, discursivas, en torno a textos para adquirir, producir y analizar conocimientos (Carlino, 2013). En el análisis de los relatos, se hace énfasis en la dimensión de enseñanza (alfabetización), más que en el aprendizaje (literacidad) y se trabaja mucho más la lectura que la escritura. Esto es así, en gran medida porque la segunda implica mayor carga de trabajo pedagógico. En este punto se plantea un reclamo extendido respecto de la falta de recursos y la masividad: "Las prácticas mejorarían con la inclusión de más actividades de producción escrita... uno de los principales problemas que percibo en este punto es el de gran matrícula que tenemos en las cátedras de los primeros años... la dificultad vinculada a que un docente pueda monitorear las producciones de 120/140 en simultáneo" (docente de Psicología). De este modo, en los relatos se asocia básicamente la 
alfabetización académica a la comprensión de textos y se disocia el trabajo entre lectura y escritura. En este sentido, es importante favorecer propuestas pedagógicas que habiliten procesos de leer y escribir no sólo para comprender y estudiar, sino también para apropiarse significativamente de los contenidos y construir conocimiento en cada campo disciplinar.

\section{RELATOS ACERCA DE LAS PRÁCTICAS DE EVALUACIÓN}

Como sabemos, las prácticas de evaluación cuando se reproducen pueden sentenciar las desigualdades en términos de rendimiento que suelen ser asumidas como naturales. En los primeros años, se encuentran estudiantes que no aprueban las diversas instancias de acreditación, situación que produce dos problemáticas: una que se expresa en el retraso académico y otra que se traduce en elevados índices de abandono. En este sentido, Ezcurra (2011) señala que, la desaprobación reiterada, al comienzo, es un factor de abandono; ello confirma que la evaluación ocupa un lugar estratégico en la construcción de estrategias de democratización, inclusión y acompañamiento de los/ as estudiantes

Para poder rastrear los discursos de los docentes sobre prácticas de evaluación en los seminarios destinamos un foro denominado "La evaluación en tiempo de pandemia", en el que les solicitamos que elaboren un pequeño texto que responda a los siguientes interrogantes: ¿Cómo se plantea la evaluación en sus cátedras?, ¿Qué adecuaciones tuvieron que hacer en tiempos de pandemia? ¿Qué mejoras consideran que pueden hacer para lograr una evaluación formativa? A continuación, se seleccionan las participaciones que fueron más recurrentes y representativas de cada uno de estos interrogantes.

¿Cómo se plantea la evaluación en sus cátedras?, ¿Qué adecuaciones tuvieron que hacer en tiempos de pandemia? ¿Qué mejoras consideran que pueden hacer para lograr una evaluación formativa?

En referencia al primer interrogante del foro, tomamos como significativo como en las prácticas docentes de evaluación, de manera global, se observó la falta de adecuación en las cátedras de pertenencia de los cursantes al nuevo escenario de entornos virtuales de enseñanza: "El parcial, se tomó vía aula web, con un horario predeterminado, dándoles 5 preguntas a responder en 12 horas, La evaluación se aprobaba con el $70 \%$ de las respuestas correctas" (docente de Ingeniería). Extrapolar las dinámicas áulicas desarrolladas en la presencialidad, a la modalidad de enseñanza en entornos virtuales fue una práctica generalizada de los docentes de ambos seminarios. En otros casos, se evidenció que: "Las materias que tienen una carga de reflexión teórica, es difícil reducirse a parámetros estandarizados para la evaluación" (docente de Psicolo- 
gía). Cuenta de ello, lo observamos en las dificultades que presentaron las modificaciones necesarias para las instancias virtuales de las evaluaciones, con la cual, queda explicitada la vinculación evaluación / enseñanza, muchas veces olvidada en la presencialidad.

En cuanto al segundo interrogante referido a los problemas y adecuaciones que presentaron las prácticas de evaluación, se vieron reflejadas principalmente por aquellas cátedras que presentan masividad en los primeros años: "La cursada virtual, trajo algunos problemas, porque al tratarse de comisiones muy numerosas más de 70 alumnos, la corrección por parte de los ayudantes, son imposible" (docente de Abogacía). Se observó que la retroalimentación en los entornos virtuales de enseñanza presentó dificultades en las cátedras masivas, impidiendo adecuaciones a las prácticas de evaluación, observándose que muchas veces quedó reducida al examen como única fuente de evidencia de aprendizaje, con dificultades en la interacción, la conversación entre docente y estudiantes, para acceder a los razonamientos y argumentos que dieron origen a determinadas respuestas. Por el contrario, la información que se produce a través de la evaluación genera conocimiento de carácter retroalimentador; es decir, significa o representa un incremento progresivo de conocimiento sobre el objeto evaluado. Desde esta perspectiva, la evaluación permite poner de manifiesto aspectos o procesos que, de otra manera permanecen ocultos, posibilita una aproximación en forma más precisa a la naturaleza de los procesos evaluados.

Con relación al tercer interrogante que exploró sobre cuestiones de mejora de la evaluación y evaluación formativa, cabe aclarar que, en ambos seminarios se orientó al trabajo de las prácticas de evaluación desde la perspectiva de la evaluación como oportunidad, tal como la trabajan Anijovich y Cappelletti (2017:15), noción que invita a que los/ las estudiantes pongan en juego sus saberes, visibilicen sus logros y aprendan a reconocer sus debilidades y fortalezas, además de cumplir la función "clásica" de aprobar, promover, certificar. Este posicionamiento sobre la evaluación de los aprendizajes permitió interrogar, reflexionar, desnaturalizar, problematizar cuestiones referidas a la evaluación que, por lo general, están implícitas en las prácticas de enseñanza y aprendizaje y dio la posibilidad a los cursante de abordar la evaluación más allá de las cuestiones de selección, control, y meritocracia: "El tema me hizo reflexionar respecto a la posibilidad de diseñar más instancias de evaluación durante el desarrollo de la cursada, y que permitan poner en juego variedad de aspectos. Esto desde un posicionamiento que no penalice, y que permita construir tales instancias como oportunidades que pongan en el centro los aprendizajes y la enseñanza" (docente de Ciencias Médicas). La reflexión que realizó el docente está en sintonía con el planteo de Pierella (2016), que la evaluación debe retroalimentar el aprendizaje y la enseñanza, aportando información a estudiantes y profesores/as, a fin de orientar el desempeño futuro de los/as primeros/as y de introducir cambios en la práctica pedagógica de los/as segundos/as.

Por último, cabe resaltar que el tema que convocó en el foro habilitó algunos interrogantes sobre los sentidos de la evaluación acerca 
de para qué y cómo evaluamos. Al mismo tiempo, permitió reflexionar cómo las prácticas de evaluación están atravesadas por los problemas de justicia y equidad.

\section{REFLEXIONES FINALES}

El COVID-19 vino a dislocar, entre tantas otras cosas, la manera de vincularnos en el espacio educativo y los modos del vínculo pedagógico. El escenario inédito y los cambios ocurridos interpelaron la enseñanza universitaria, de modo que fueron surgiendo nuevas representaciones de los espacios formativos, nuevos sentidos y entramados de las prácticas pedagógicas. En términos de prácticas docentes, hizo re-pensar la planificación de la enseñanza para ser desarrollada en entornos virtuales, la transmisión de los contenidos y la alfabetización académica, la evaluación, entre otros aspectos.

En tal sentido, los Seminarios estuvieron orientados a ofrecer herramientas conceptuales y metodológicas para indagar y desnaturalizar sus propias prácticas para construir un saber pedagógico que contribuya con la mejora de las trayectorias estudiantiles en el ingreso y los primeros años, y así garantizar el ejercicio efectivo del derecho que los asiste. Los discursos y relatos producidos por las y los cursantes en esos espacios curriculares resultan un insumo muy valioso para reflexionar y repensar las prácticas docentes, y abonar a la consolidación de una pedagogía universitaria basada en un enfoque de derechos.

En este trabajo quisimos reseñar las problematizaciones en torno a las mejores condiciones pedagógicas y didácticas para que las/os estudiantes aprendieran en el contexto de la virtualidad, y a repensar los procesos de apropiación del conocimiento y los modos de articular la especificidad de los saberes disciplinares con los entornos virtuales. Cabe señalar el impacto profundo que tuvo la experiencia de la virtualización de la enseñanza en la docencia universitaria. Para algunos docentes, los cambios introducidos en la propuesta pedagógica tendrán efectos perdurables en los modos de enseñar, incluso en el retorno a la presencialidad.

\section{El escenario inédito y los cambios ocurridos interpelaron la en- señanza universitaria, de modo que fueron surgiendo nuevas representaciones de los espacios formativos, nuevos sentidos y entramados de las prácticas pedagógicas.}




\section{BIBLIOGRAFÍA}

Anijovich, R. y Cappelletti, G. (2017). “La evaluación como oportunidad”. Buenos Aires: Paidós.

Carlino, P. (2013). "Alfabetización académica diez años después". Revista Mexicana de Investigación Educativa, vol. 18, núm. 57, 2013, pp. 355-381. Consejo Mexicano de Investigación Educativa, A.C. Distrito Federal, México.

Ezcurra, A. M. (2003). "Principales dificultades de los alumnos de primer ingreso al grado". Los Polvorines. UNGS.

Gómez, P. A. (2020). “Módulo 3: Cultura de la conectividad y subjetividades actuales en la relación estudiantes - docentes". Pedagogía crítica y didáctica en la enseñanza digital. Buenos Aires: Ministerio de Educación de la Nación.

Juarros, M.F. \& Levy, E. (2020). “Módulo 1: La práctica docente en la educación a distancia. La relación pedagógica mediada por tecnologías". Pedagogía crítica y didáctica en la enseñanza digital. Buenos Aires: Ministerio de Educación de la Nación.

Pierella, M. P. (2016). "Los exámenes en el primer año de la universidad. ¿Instancias de formación o mecanismos de selectividad social?" Trayectorias universitarias, vol. 2 pp. 11-19, La Plata: UNLP. 